\title{
DCBWL : A New Channel Borrowing Scheme for Mobile Cellular Communication Systems
}

\author{
TEJASKUMAR PATEL, DINESH K. ANVEKAR and B. S. SONDE \\ Department of Electrical Communication Engineering \\ Indian Institute of Science, Bangalore 560012, India. \\ E-mail: dka@ece.iisc.ernet.in
}

\begin{abstract}
A new channel borrowing scheme which allows each cell to lend its channels to its adjacent cells without locking them in nearby co-channel cells is presented. Performance of the new scheme is compared with that of a channel borrowing without locking (CBWL) scheme by computer simulation of a 49-cell model of a mobile cellular system. It is shown by simulation results that the new scheme has much lower call blocking probability as compared to the CBWL scheme, for both uniform and hot-spot call traffic patterns.
\end{abstract}

\section{Introduction}

During the past decade several algorithms and schemes have been proposed to address the problems of handover management and channel assignment, keeping in view the constraint of limited frequency spectrum resources. One of the solutions to meet the increasing demand on the finite frequency spectrum resource is to employ dynamic channel allocation (DCA) schemes with provision for borrowing channels from neighbouring cells. Several algorithms for DCA [1]- [4], and for channel borrowing [4]-[7] have been proposed. In conventional DCA schemes, no channel is assigned to any cell permanently but all the channels are kept in a single pool, and based on request they are assigned to new call/handoff requests on the basis of minimum allowed reuse distance and on the basis of some channel allocation strategy as in [2] and [4]. On termination of calls, the assigned channels are switched to optimize the separation between co-channel coverage areas as in [1]. Among the recent DCA schemes, the scheme proposed in [3], attempts to dynamically keep co-channel size of any channel to its compact pattern while assigning channels. A DCA scheme involving channel borrowing without locking (CBWL) has been proposed in [5]-[7]. In this scheme, channel borrowing is allowed from neighbouring cell's group of channels which is dedicated for borrowing from the current cell without locking it in the nearby co-channel cells. Here, the borrowed channels are used with lower power levels and within a limited area to maintain acceptable signal to interference ratio (SIR). In this paper, a new scheme called directional channel borrowing without locking (DCBWL) is proposed as an improvement over the CBWL scheme.

\section{The DCBWL Scheme}

Consider a basic hexagonal layout of a cellular communication system with 7 cell reuse pattern as shown in Fig. 1. Here, the channels of a cell are divided into seven distinct groups. The channels of group zero are reserved for exclusive use within the given cell while the other six groups of channels can be lent to adjacent cells. In the CBWL scheme, the $i^{\text {th }}$ adjacent cell can only borrow channels from the $i^{t h}$ group. Thus, for example, one specific group of channels is allowed to be borrowed only by cell $B_{0}$ maintaining minimum reuse distance $N 1$ between two co-channel cells to be 3 units with a single worst case interferer condition as in Fig. 1. Here, the circled portions are areas where possibly the same channels can be reused. However, in one such cluster, at any time, a particular channel can be used only in one cell. The shaded portions in Fig. 1 show an example of the single worst case interferer condition. Now, as shown in Fig. 2, if that specific group of channels is allowed to be borrowed by cell $B_{0}$ as well as cell $G_{0}$, it results in the same single worst case interferer situation (with minimum reuse distance $N 1=3$ ) as in the previous case. This shows that for the same SIR as with the CBWL scheme, now the same channel group can be allowed to be borrowed by two neighboring cells as compared to just one in the case of CBWL scheme. This observation is the motivation for the design of the DCBWL scheme. As the DCBWL scheme has effectively twice the number of channels that can be borrowed from a neighboring cell, it results in performance improvement over CBWL scheme under both normal load and hot-spot conditions. The channel grouping and borrowing policy under DCBWL scheme is such that channels from a group of borrowable channels are allowed to be borrowed only by two neighboring cells which are themselves adjacent to each other. For example, the groups of borrowable channels in cell $\mathrm{A}$ are $a_{B C}, a_{C D}, a_{D E}, a_{E F}, a_{F G}$ and $a_{G B}$. Here the subscripts indicate the two neighboring cells which can borrow channels from the particular group. 


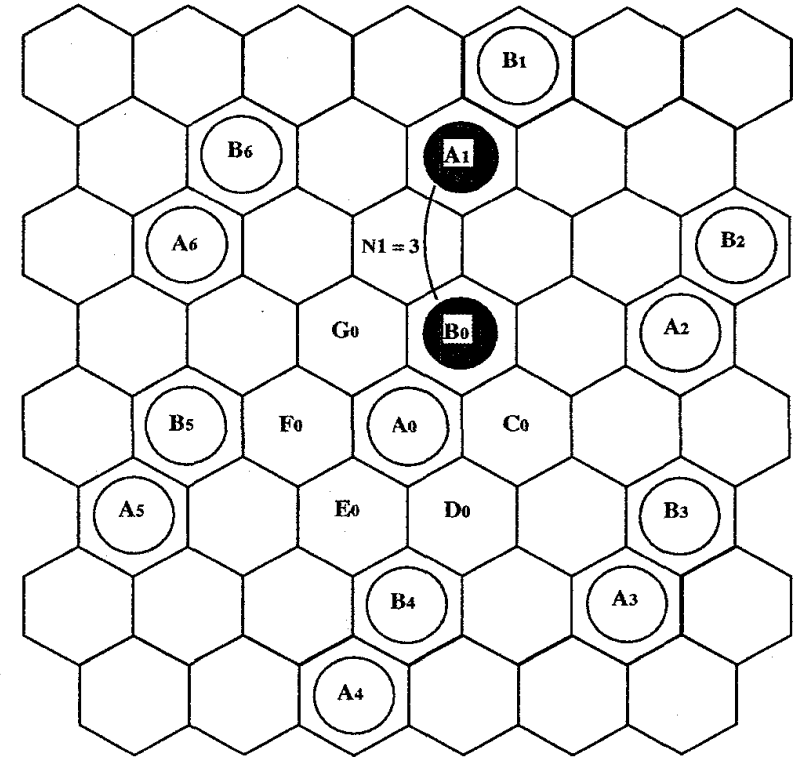

Figure 1: Cochannel cells in single worst case interferer condition for CBWL with $\mathrm{N} 1=3$

Thus, $a_{B C}$ group of channels can be borrowed by cells $\mathrm{B}$ and $\mathrm{C}, a_{C D}$ group of channels by cell $\mathrm{C}$ and $\mathrm{D}$, and so on.

In the DCBWL scheme, as a channel can be borrowed without locking it in the nearby co-channel cells, the distance between two cells using the same channel is reduced. Therefore, the carrier power must be reduced for a borrowed channel to maintain the same SIR as for regular channels. This restricts the usage of borrowed channels to a smaller coverage area. Because of this restriction, the mobiles in the system are divided into two types: (i) Type A mobiles which can borrow channels and (ii) Type B mobiles that are not permitted to borrow channels. With this division of mobiles, the DCBWL scheme can be applied under two cases which are distinguished based on whether channel rearrangement between type A and type $\mathrm{B}$ mobiles is allowed or not. The two cases are called here as DCBWL with Channel Rearrangement (DCBWL/CR), and DCBWL with No Rearrangement of channels (DCBWL/NR). When a new call needs a channel in a celt, it is assigned_a channel, if available, from within the cell. However, if free channels are not available, then an attempt is made to borrow a channel from the neighbouring cells. The procedure for channel allocation to be used for a borrowed channel will depend on whether DCBWL/NR or DCBWL/CR procedure is used.

\subsection{DCBWL/NR Procedure}

In DCBWL/NR, no rearrangement is made between channels of type A and type B mobiles at the time of channel

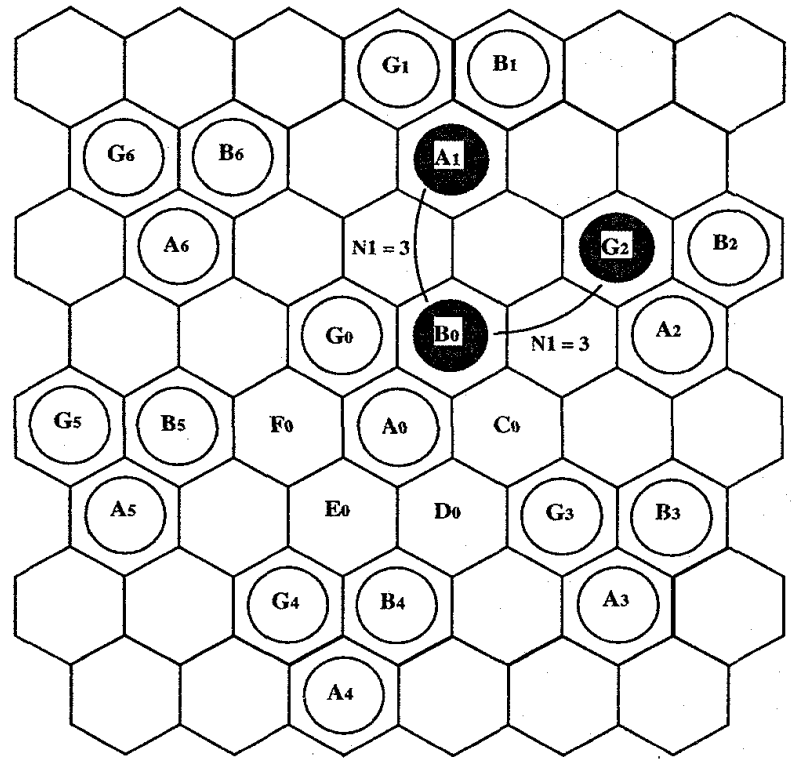

Figure 2: Cochannel cells in single worst case interferer condition for DCBWL with $\mathrm{N} 1=3$

allocation. If a cell finds that all of its channels are used and if a type A mobile needs a channel, then an attempt is made to borrow a channel from its neighbour. For this, the neighbouring cells are checked one after another to find a borrowable channel. If a channel is available, it is assigned to the mobile, otherwise the call is blocked. However, if a call request is from a type $B$ mobile user, then it is just blocked.

\subsection{DCBWL/CR Procedure}

In DCBWL/CR, channel rearrangement is allowed between type A and type B mobile users to optimize the utilization of borrowable channels. Here, among the ongoing calls within the cell, distinction is made between type A mobile and type B mobile calls. For a type A mobile, if all the channels in the cell are used, an attempt is made for borrowing a channel from a neighbouring cell as in the DCBWL/NR case. However, if a call request is from a type B mobile user, and if a borrowable channel is available, then the borrowed channel is assigned to a type A mobile user, and the regular channel of the type A mobile user is allocated to the type B mobile user. Thus, the benefit of borrowing channels is extended to type B mobile users also.

\subsection{DCBWL with Channel Shuffling}

Channel shuffling can be used with the DCBWL scheme to improve channel utilization efficiency and further reduce 
call blocking probability. Without channel shuffling, when a cell finds that there are no borrowable channels in its neighbors, it just blocks a call request. However, if the cell has lent any channels to its neighbor which has at that time a non-borrowable channel available, then the call on the borrowed channel can be switched to the non-borrowable channel. With this call switching, the borrowed channel becomes available in the owner cell for assignment to the new call. Channel shuffling is also allowed in the case when neighbor does not have a non-borrowable channel available but it is in the position to borrow a channel from its neighbors.

\section{Comparison of DCBWL with Other Schemes}

As compared to DCA schemes, DCBWL offers advantages in terms of hardware simplicity of the systems as neither dynamic power control is needed nor each base station (BS) is required to be capable of tuning to all the channels used in the specific layout. Channel reuse distance in DCBWL can also be kept at a desired minimum. Further, the control and management of channel assignment under DCBWL is much simplified as no global information on channels used is required. As compared to fixed channel allocation (FCA) schemes, DCBWL always results in better performance due to borrowing of channels without SIR degradation. The performance of DCBWL can also be expected to be better than CBWL as the number of channels that can be borrowed by each cell is effectively doubled. However, the BSs in the case of DCBWL are required to be capable of tuning to some more channels. This leads to slightly higher cost of BS tranceivers with DCBWL as compared to CBWL.

\section{Co-channel Interference DCBWL}

in

An analysis of co-channel interference for the DCBWL scheme is given in this section. Consider a cellular system with $R$ as cell radius and $D$ as the distance between nearest co-channel cells. The cluster size $N$ is given by $N=i^{2}+i j+j^{2}$ where $i$ and $j$ are the shift parameters which determine the reuse pattern and co-channel size. With the regular hexagonal layout, the ratio $D / R$ is given by $D / R$ $=\sqrt{3 N}$.

In the following analysis, only a single worst case interferer in FCA and DCBWL is considered, and the schemes are compared without considering fading as it will affect both the cases in the same way. The other co-channel cells may also affect SIR but the degradation for both cases will be by the same factor.

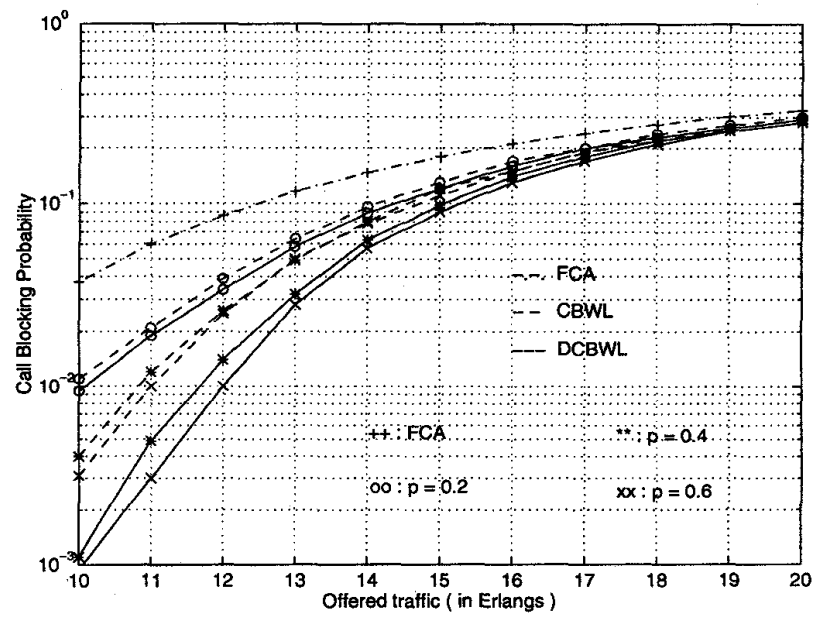

Figure 3: Call blocking probabilities with channel rearrangement

In FCA under worst case, a mobile will be at a distance $R$ from its BS while $D-R$ from the nearest BS using the same channel which is responsible for interference. Thus, SIR in this case is given by

$$
S I R_{0}=\left(\frac{D-R}{R}\right)^{\gamma}=(\sqrt{3 N}-1)^{\gamma}
$$

where $\gamma$ is a propagation constant.

Consider the single worst case interferer condition under DCBWL as shown in Fig. 2. Here, $A_{0}$ and $A_{1}$ are cochannel cells, and it is assumed that $B_{0}$ has borrowed a channel from $A_{0}$. Under this single worst case interferer condition, the two cases possible are:

Case 1: $A_{1}$ is using the same channel;

Case 2: $G_{2}$ has borrowed the same channel from $A_{2}$.

An analysis for these two cases is as follows.

Case 1: With $i$ and $j$ as shift parameters, minimum reuse factor in this case is given as $N 1=$ $\min \left[i^{2}+i(j-1)+(j-1)^{2},(i-1)^{2}+(i-1) j+j^{2}\right]$

and minimum co-channel distance is $D 1=\sqrt{3 N 1} R$. The co-channel interference analysis for this case is the same as for the CBWL scheme [5]. For this case it can be shown [5] that to have no performance degradation

$$
\frac{r}{R}=\frac{\sqrt{3 N 1}(\sqrt{3 N 1}-1)}{(\sqrt{3 N}-1)^{2}+\sqrt{3 N 1}-1}
$$

and

$$
p \simeq 1.2\left(\frac{r}{R}\right)^{2}
$$

where $r$ is the maximum radius from the cell center upto which a borrowed channel can be used, and $p$ is the ratio 


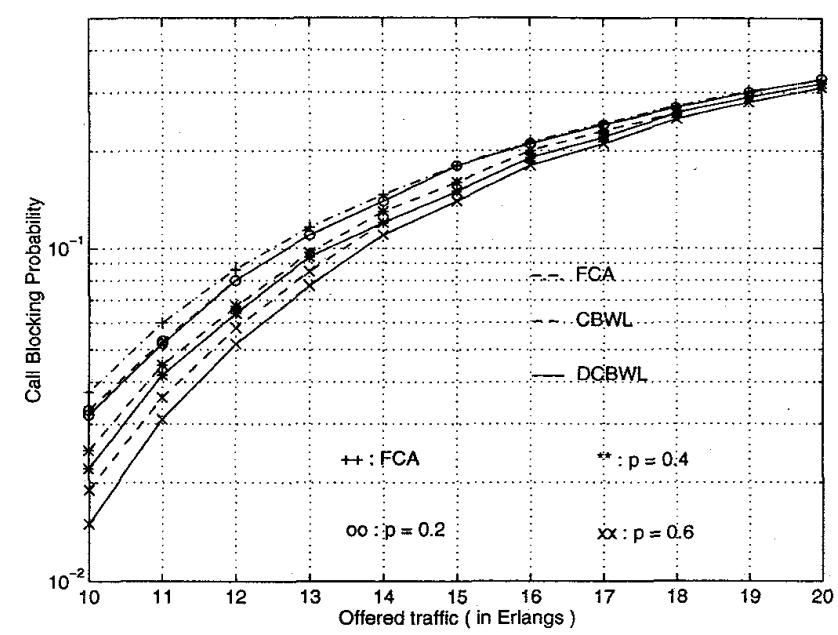

Figure 4: Call blocking probabilities without channel rearrangement

of the area of the inner circle of radius $r$ and the area of a cell.

Case 2: Under this case, co-channel interference is between two cells in both of which the interfering channels are borrowed from the neighbouring cells. An example situation for this case is shown in Fig. 2. Here, $B_{0}$ has borrowed a channel from $A_{0}$ and $G_{2}$ has borrowed the co-channel from $A_{2}$. Being a borrowed channel in both the cells, it is used with lower power and limited coverage. In the worst case, the distance between a mobile and the BS under consideration is $r$, and $D 1-r$ is the distance of the mobile from the nearest BS using the same channel. Thus, SIR for this case is

$$
S I R_{1}=\left(\frac{D 1-r}{r}\right)^{\gamma}=\left(\frac{D 1}{r}-1\right)^{\gamma}
$$

As in Case 1, to have no performance degradation, $S I R_{1} \geq$ $S I R_{0}$. Equating $S I R_{1}$ to $S I R_{0}$ gives

$$
\frac{r}{R}=\sqrt{\frac{N 1}{N}}
$$

and

$$
p=1.2\left(\frac{N 1}{N}\right)
$$

For $N=7, N 1=3$, under Case $2, p=0.51$, while under Case $1, p=0.19$. Therefore, Case 1 puts a greater restriction on the usage of borrowed channels as compared to Case 2. This indicates that to maintain the same SIR as in FCA case, the usage of borrowed channels in DCBWL has to be restricted to the fraction of area as given by $p$ of Case 1.

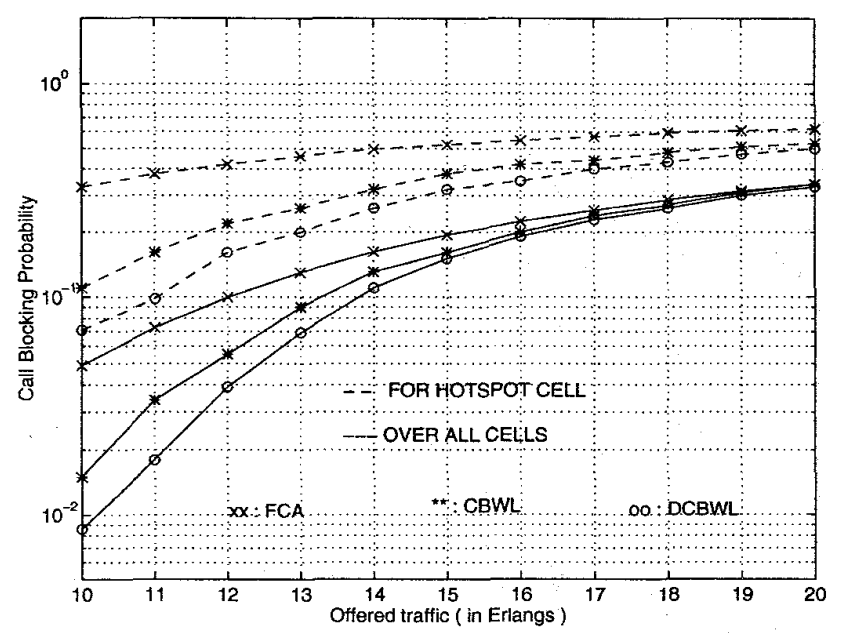

Figure 5: Call blocking probabilities for hot-spot situation with channel rearrangement

\section{Simulation Model and Results}

The performance of the DCBWL scheme with respect to call blocking probability has been evaluated by computer simulation of a cellular system model with 49 cells arranged in a $7 \times 7$ layout. Each cell in the model has 15 channels of which three channels are reserved solely for use within the cell. The other 12 channels are divided into six groups each with two channels. A restriction imposed on channel borrowing is that at any time a maximum of 10 channels are allowed to be borrowed by the neighbouring cells. The call arrivals in the cells are assumed to follow the Poisson distribution and the call durations are exponentially distributed with a mean of 60 seconds. The two call traffic pattern considered for simulation were (i) Uniform traffic in every cell and (ii) Hot-spot traffic with one cell having double the offered traffic as in the other cells. The simulation runs were continued for 60,000 calls in each case and the statistics were collected after the first 5000 calls to eliminate the initial transient phase of the system. Also, the results were evaluated for the nine middle cells of the system to avoid the edge effects on simulation results.

The simulation results are shown in Figs 3-7. As seen from Figs 3-5, the DCBWL scheme has much lower call blocking probability as compared to the CBWL scheme, for both the cases with and without channel rearrangement. For example, with an offered traffic of 14 Erlangs, the new scheme has $16.9 \%$ improvement in performance in terms of call blocking probability for the case without channel rearrangement, and $39.1 \%$ improvement with channel rearrangement. These figures are $25 \%$ and $46 \%$ respectively for the case with a hot-spot cell which has twice the offered call traffic as in normal cell, and $p=0.4$. These results clearly show the superiority of DCBWL over CBWL. 


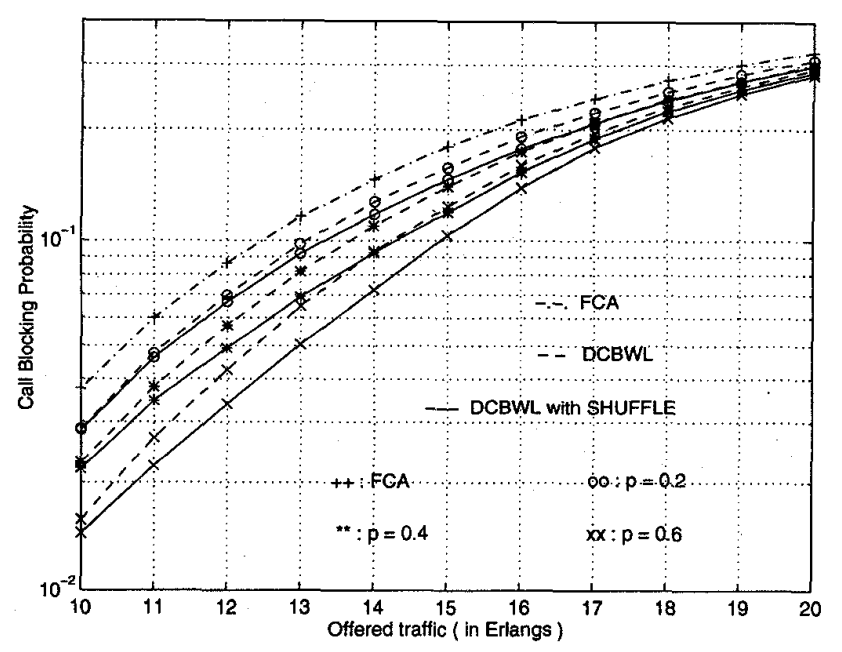

Figure 6: Call blocking probabilities for the DCBWL/NR scheme with Channel Shuffling

Figs 6 and 7 show the effect of channel shuffling. As seen here, channel shuffling when used with the DCBWL scheme further reduces call blocking probability.

\section{Conclusion}

The DCBWL scheme presented in this paper results in lower call blocking probability as compared to FCA and the CBWL schemes without degrading SIR of the system under both normal load and hot-spot call traffic conditions. The performance improvement is possible with the DCBWL scheme because a single group of channels is allowed to be borrowed by two neighboring cells instead of only one as in the CBWL scheme. The implementation of the DCBWL scheme is as simple as the CBWL scheme. Channel management under the DCBWL scheme is also much simplified as compared to conventional DCA schemes which need to maintain global information about channel allocations. However, the complexity of tranceivers in the BSs is slightly higher than those with the CBWL scheme, as they must now be capable of tuning to the additional borrowed channels also.

\section{References}

[1] D. C. Cox and D. O. Reudink," Increasing channel occupancy in large-scale mobile radio systems: Dynamic channel reassignment," IEEE Trans. Veh. Technol., vol. 22, pp. 218-223, Nov. 1973.

[2] S. M. Elnoubi , R. Singh and S. C. Gupta," A new frequency channel assignment in high capacity mobile com-

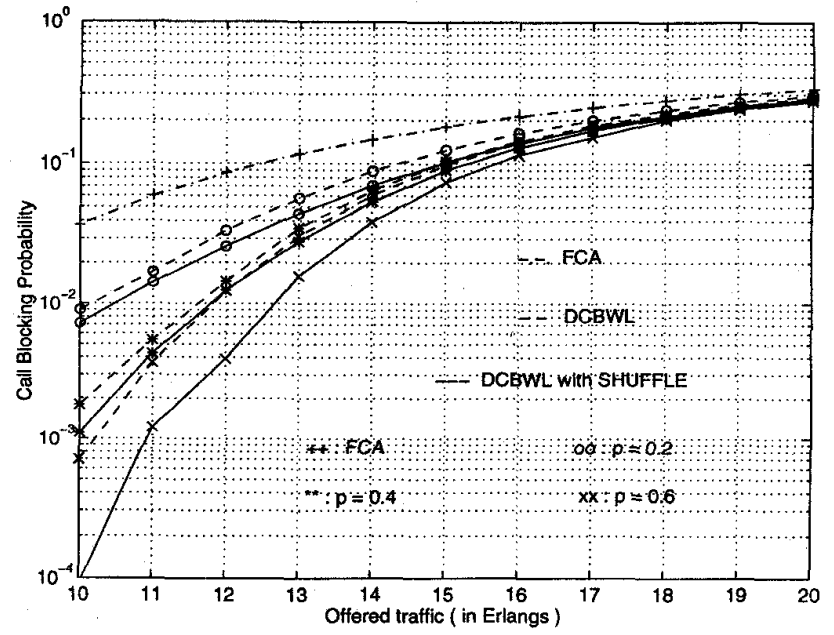

Figure 7: Call blocking probabilities for the DCBWL/CR scheme with Channel Shuffling

munication systems," IEEE Trans. Veh. Technol. , vol. 31, no. 3, pp. 125-131, Aug. 1982.

[3] K. L. Yeung and T. P. Yum," Compact Pattern Based Dynamic Channel Assignment for Cellular Mobile Systems," IEEE Trans. Veh. Technol. , vol. 43, no. 4, pp. 892-896, Nov. 1994.

[4] M. Zhang and T. P. Yum," Comparisions of channel assignment strategies in cellular mobile telephone systems," IEEE Trans. Veh. Technol. , vol. 38, pp. 211-215, Nov. 1989.

[5] H. Jiang and S. S. Rappaport," CBWL: A new channel assignment and sharing method for cellular communication systems," IEEE Trans. Veh. Technol., vol. 43, no. 2, pp. 313-322, May. 1994.

[6] H. Jiang and S. S. Rappaport," CBWL for sectorized cellular communication," IEEE Trans. Veh. Technol., vol. 43, no. 4, pp. 1067-1077, Nov. 1994.

[7] H. Jiang and S. S. Rappaport," Prioritized channel borrowing without locking: A channel sharing strategy for cellular communication systems," in Proc. IEEE Global Communication Conf. , Houston, TX, Nov. 29-Dec. 2, 1993, pp. 276-280. 